\title{
FIXED POINT RESULTS FOR $\omega$-INTERPOLATIVE CHATTERJEA TYPE CONTRACTION IN QUASI-PARTIAL B-METRIC SPACE
}

\section{PRAGATI GAUTAM ${ }^{1, *}$, SWAPNIL VERMA ${ }^{1}$, MANUEL DE LA SEN ${ }^{2}$, SEJAL SUNDRIYAL ${ }^{1}$}

${ }^{1}$ Department of Mathematics, Kamala Nehru College, University of Delhi, August Kranti Marg, New Delhi 110049 , India

${ }^{2}$ Institute of Research and Development of Processes, University of Basque Country, Campus of Leioa(Bizkaia)-Aptdo, 644-Bilbao, Bilbao, 48080, Spain

${ }^{*}$ Corresponding author: pgautam@knc.du.ac.in

\begin{abstract}
The purpose of this paper is to revisit Chatterjea type contraction and determine some fixed point results for interpolative Chatterjea type contraction mapping in the setting of quasi-partial b-metric space using the concept of $\omega$-admissibility introduced by Popescu. Also we present some useful examples to elucidate relevance of the concept.
\end{abstract}

\section{INTRODUCTION}

In the diversified field of non-linear analysis, Banach [1] contraction principle holds a significant position. The fixed point theorems are used to demonstrate the uniqueness of a solution of differential equations, Fredholm integral equations and Picard theorem etc. Forging ahead Banach's approach, many celebrated authors [2-7] introduced distinctive concepts. In the year 1972, Chatterjea [8] inaugurated his contraction defined as

Received February $7^{\text {th }}, 2021$; accepted March $1^{\text {st }}, 2021$; published March $17^{\text {th }}, 2021$.

2010 Mathematics Subject Classification. 46T99, 47H10, 54H25.

Key words and phrases. quasi-partial b-metric space; fixed point; $\omega$ admissible; interpolation; Chatterjea contraction.

(C)2021 Authors retain the copyrights of their papers, and all open access articles are distributed under the terms of the Creative Commons Attribution License. 
Let (X, d) be a complete metric space. A self mapping $H: X \rightarrow \mathrm{X}$ is called Chatterjea type contraction if

$$
d(H \sigma, H \theta) \leq \delta[d(\sigma, H \theta)+d(\theta, H \sigma)]
$$

for all $\sigma, \theta \in X$, where $\delta \in(0,1 / 2)$. Then he interestingly proved that it has a unique fixed point in complete metric space. Additionally this result was proved by Mishra et al. [9] in complete quasi-partial b-metric space for interpolative Chatterjea type contraction, which can be designated as,

Let $\left(\mathrm{X}, q p_{b}\right)$ be a complete quasi-partial b-metric space. A self mapping $H: X \rightarrow \mathrm{X}$ is called interpolative Chatterjea type contraction if there exists $\delta \in\left[0, \frac{1}{s}\right), \rho \in(0,1)$ such that

$$
q p_{b}(H \sigma, H \theta) \leq \delta\left[q p_{b}(\sigma, H \theta)\right]^{\rho}\left[\frac{1}{s^{2}} q p_{b}(\theta, H \sigma)\right]^{1-\rho}
$$

for all $\sigma, \theta \in X$.

Afterwards as a modification in the concept of $\alpha$-admissible maps, Popescu [10] introduced $\omega$-orbital admissible maps.

In this research field, several authors [13-15] have made valuable contributions. In this paper, we commence the concept of $\omega$-interpolative Chatterjea contraction in quasi-partial b-metric space and deliver relevant examples.

\section{Preliminaries}

Definition $2.1[10]$ : Let $\omega: X \times X \rightarrow[0, \infty)$ be a mapping and $X \neq \phi$. A self mapping $H: X \rightarrow X$ is called $\omega$-admissible if,

$$
\omega(\sigma, H \sigma) \geq 1 \Longrightarrow \omega\left(H \sigma, H^{2} \sigma\right) \geq 1
$$

for all $\sigma \in X$.

In order to ignore the continuity of contractive mappings, we often consider the following condition.

(M) If we take a sequence $\theta_{n}$ in $\mathrm{X}$ such that $\omega\left(\theta_{n}, \theta_{n+1}\right) \geq 1$, for all n. Also as $n \rightarrow \infty, \theta_{n} \rightarrow \theta \in X$, then from $\theta_{n}$ there exists $\theta_{n(k)}$ such that for all $\mathrm{k}, \omega\left(\theta_{n(k)}, \theta\right) \geq 1$.

Definition 2.2 [11] : A quasi-partial metric space on a set non-empty set $\mathrm{X}$ is a function $q p: X \times X \rightarrow[0, \infty)$ that satisfies the following properties:

$\left[Q P_{1}\right]$ If $q p(\sigma, \sigma)=q p(\sigma, \theta)=q p(\theta, \theta)$ then $\sigma=\theta$

$\left[Q P_{2}\right] q p(\sigma, \sigma) \leq q p(\sigma, \theta)$

$\left[Q P_{3}\right] q p(\sigma, \sigma) \leq q p(\theta, \sigma)$ 
$\left[Q P_{4}\right] q p(\sigma, \theta)+q p(\delta, \delta) \leq q p(\sigma, \delta)+q p(\delta, \theta)$ for all $\sigma, \theta, \delta \in X$

Definition $2.3[12]$ : A quasi-partial b-metric space on a set $X \neq \phi$ is a function $q p_{b}: X \times X \rightarrow[0, \infty)$ such that for a real number $s \geq 1$ satisfies the following properties:

$\left[Q P b_{1}\right]$ If $q p_{b}(\sigma, \sigma)=q p_{b}(\theta, \theta)=q p_{b}(\sigma, \theta)$ then $\sigma=\theta$

$\left[Q P b_{2}\right] q p_{b}(\sigma, \sigma) \leq q p_{b}(\sigma, \theta)$

$\left[Q P b_{3}\right] q p_{b}(\sigma, \sigma) \leq q p_{b}(\theta, \sigma)$

$\left[Q P b_{4}\right] q p_{b}(\sigma, \theta) \leq s\left[q p_{b}(\sigma, \delta)+q p_{b}(\delta, \theta)\right]-q p_{b}(\delta, \delta)$ for all $\sigma, \theta, \delta \in X$.

Here, we present an example to show the usability of the concept.

Example 2.1: Let $\mathrm{X}=\left[0, \frac{\pi}{2 k}\right]$. Define $q p_{b}(\sigma, \theta)=\sin k\left|\sigma^{2}-\theta^{2}\right|$, where $k \geq 1$

Here, $q p_{b}(\sigma, \sigma)=\sin k\left|\sigma^{2}-\sigma^{2}\right|=0=q p_{b}(\theta, \theta), q p_{b}(\sigma, \theta)=\sin k\left|\sigma^{2}-\theta^{2}\right|$

then $0=\sin k\left|\sigma^{2}-\theta^{2}\right| \Longrightarrow \sigma=\theta$ i.e $\left[Q P b_{1}\right]$ satisfied.

$q p_{b}(\sigma, \sigma)=0 \leq q p_{b}(\sigma, \theta)$ as $0 \leq \sin k\left|\sigma^{2}-\theta^{2}\right|$ i.e $\left[Q P b_{2}\right]$ satisfied.

$q p_{b}(\sigma, \sigma)=0 \leq q p_{b}(\theta, \sigma)$ as $0 \leq \sin k\left|\theta^{2}-\sigma^{2}\right|$ i.e $\left[Q P b_{3}\right]$ satisfied.

As $\sigma, \theta, \delta \in X$

$$
\begin{gathered}
\left|\sigma^{2}-\theta^{2}\right| \leq \frac{\pi}{2 k},\left|\sigma^{2}-\theta^{2}\right|+\left|\delta^{2}-\theta^{2}\right| \leq \frac{\pi}{2 k} \\
k\left[\left|\sigma^{2}-\delta^{2}\right|+\left|\delta^{2}-\theta^{2}\right|\right] \leq \frac{\pi}{2},
\end{gathered}
$$

Since sin k is increasing.

$q p_{b}(\sigma, \theta)+q p_{b}(\delta, \delta)=\sin k\left|\sigma^{2}-\theta^{2}\right|$

$$
\begin{gathered}
\leq \operatorname{sink}\left(\left|\sigma^{2}-\delta^{2}\right|+\left|\delta^{2}-\theta^{2}\right|\right) \leq k\left(\left|\sigma^{2}-\delta^{2}\right|+\left|\delta^{2}-\theta^{2}\right|\right) \\
\leq k\left(\sin k\left(\left|\sigma^{2}-\delta^{2}\right|+\left|\delta^{2}-\theta^{2}\right|\right)\right) \leq k\left[q p_{b}(\sigma, \delta)+q p_{b}(\delta, \theta)\right] \\
\leq s\left[q p_{b}(\sigma, \delta)+q p_{b}(\delta, \theta)\right]
\end{gathered}
$$

where, $s \geq k \geq 1$ i.e. $\left[\mathrm{QPb}_{4}\right]$ satisfied.

Therefore, $\left(X, q p_{b}\right)$ is a quasi-partial-b metric space. 


\section{MAin RESUlts}

Here, we introduce the concept of $\omega$-interpolative Chatterjea type contractions in quasi-partial b-metric space.

Definition 3.1: Let $\left(\mathrm{X}, q p_{b}\right)$ be a complete quasi-partial b-metric space. A self mapping $\mathrm{H}: \mathrm{X} \rightarrow \mathrm{X}$ is called $\omega$-interpolative Chatterjea type contraction if there exists $\delta \in\left[0, \frac{1}{s}\right), \rho \in(0,1)$ such that

$$
\omega(\sigma, \theta) q p_{b}(H \sigma, H \theta) \leq \delta\left[q p_{b}(\sigma, H \theta)\right]^{\rho}\left[\frac{1}{s^{2}} q p_{b}(\theta, H \sigma)\right]^{1-\rho}
$$

for all $\sigma, \theta \in \mathrm{X}$.

Our main result is as follows:

Theorem 3.1. Let $H: X \rightarrow X$ be an $\omega$-admissible self mapping which forms $\omega$-interpolative Chatterjea type contraction on a complete quasi-partial b-metric space $\left(X, q p_{b}\right)$. If there exists $\sigma_{0} \in X$ such that $\omega\left(\sigma_{0}, H \sigma_{0}\right) \geq 1$, then $H$ has a fixed point in $X$.

Proof: Let $\sigma_{0} \in\left(X, q p_{b}\right)$ such that $\omega\left(\sigma_{0}, H \sigma_{0}\right) \geq 1$.

Let us consider a sequence $\sigma_{n}$ defined as $\sigma_{n}=H^{n}\left(\sigma_{0}\right), n \geq 0$.

Considering for some $n_{0}$,

If $\sigma_{n_{0}}=\sigma_{n_{0}+1}$, this implies that $\sigma_{n_{0}}$ is a fixed point of $H$.

If $\sigma_{n_{0}} \neq \sigma_{n_{0}+1}$, for all $n \geq 0$ then,

$$
q p_{b}\left(\sigma_{n}, H \sigma_{n}\right)=q p_{b}\left(\sigma_{n}, H \sigma_{n+1}\right)>0
$$

Also $H$ is $\omega$-admissible, $\omega\left(\sigma_{1}, \sigma_{2}\right)=\omega\left(H \sigma_{0}, H \sigma_{1}\right) \geq 1$.

$\Longrightarrow \omega\left(\sigma_{n}, \sigma_{n+1}\right) \geq 1$, for all $n \geq 0$.

Taking $\sigma=\sigma_{n}$ and $\theta=\sigma_{n-1}$, we get

$$
\begin{gathered}
q p_{b}\left(\sigma_{n+1}, \sigma_{n}\right) \leq \omega\left(\sigma_{n}, \sigma_{n+1}\right) q p_{b}\left(H \sigma_{n}, H \sigma_{n-1}\right) \\
\leq \delta\left[q p_{b}\left(\sigma_{n}, H \sigma_{n-1}\right)\right]^{\rho}\left[\frac{1}{s^{2}} q p_{b}\left(\sigma_{n-1}, H \sigma_{n}\right)\right]^{1-\rho} \\
\leq \delta\left[q p_{b}\left(\sigma_{n}, \sigma_{n}\right)\right]^{\rho}\left[\frac{1}{s^{2}} q p_{b}\left(\sigma_{n-1}, \sigma_{n+1}\right)\right]^{1-\rho} \\
\leq \delta\left[q p_{b}\left(\sigma_{n+1}, \sigma_{n}\right)\right]^{\rho}\left[\frac{1}{s^{2}}\left[s\left[q p_{b}\left(\sigma_{n-1}, \sigma_{n}\right)+q p_{b}\left(\sigma_{n}, \sigma_{n+1}\right)\right]-q p_{b}\left(\sigma_{n}, \sigma_{n}\right)\right]\right]^{1-\rho} \\
\leq \delta\left[q p_{b}\left(\sigma_{n+1}, \sigma_{n}\right)\right]^{\rho}\left[\frac{s}{s^{2}}\left[q p_{b}\left(\sigma_{n-1}, \sigma_{n}\right)+q p_{b}\left(\sigma_{n}, \sigma_{n+1}\right)\right]\right]^{1-\rho} \\
\leq \delta\left[q p_{b}\left(\sigma_{n+1}, \sigma_{n}\right)\right]^{\rho}\left[\frac{1}{s}\left[q p_{b}\left(\sigma_{n-1}, \sigma_{n}\right)+q p_{b}\left(\sigma_{n}, \sigma_{n+1}\right)\right]\right]^{1-\rho}
\end{gathered}
$$


If $q p_{b}\left(\sigma_{n-1}, \sigma_{n}\right) \leq q p_{b}\left(\sigma_{n}, \sigma_{n+1}\right)$ for all $n \geq 1$, then

$$
\frac{1}{s}\left[q p_{b}\left(\sigma_{n-1}, \sigma_{n}\right)+q p_{b}\left(\sigma_{n}, \sigma_{n+1}\right)\right]^{1-\rho} \leq q p_{b}\left(\sigma_{n}, \sigma_{n+1}\right)^{1-\rho}
$$

that is,

$$
\frac{1}{s}\left[q p_{b}\left(\sigma_{n-1}, \sigma_{n}\right)+q p_{b}\left(\sigma_{n}, \sigma_{n+1}\right)\right] \leq q p_{b}\left(\sigma_{n}, \sigma_{n+1}\right)
$$

but $q p_{b}\left(\sigma_{n+1}, \sigma_{n}\right) \leq q p_{b}\left(\sigma_{n-1}, \sigma_{n}\right)$, which is a contradiction as per equation 3.1.

Thus $q p_{b}\left(\sigma_{n-1}, \sigma_{n}\right)$ is a decreasing sequence.

Now, let $\lim _{n \rightarrow \infty} q p_{b}\left(\sigma_{n-1}, \sigma_{n}\right)=L$.

As we have, from equation 3.1

$$
\begin{gathered}
q p_{b}\left(\sigma_{n+1}, \sigma_{n}\right) \leq \omega\left(\sigma_{n}, \sigma_{n+1}\right) q p_{b}\left(H \sigma_{n}, H \sigma_{n-1}\right) \\
\leq \delta\left[q p_{b}\left(\sigma_{n+1}, \sigma_{n}\right)\right]^{\rho}\left[\frac{1}{s^{2}} q p_{b}\left(\sigma_{n-1}, \sigma_{n}\right)\right]^{1-\rho} \\
\omega(\sigma, \theta) q p_{b}\left(\sigma_{n+1}, \sigma_{n}\right)^{1-\rho} \leq \delta\left[\left[\frac{1}{s^{2}} q p_{b}\left(\sigma_{n-1}, \sigma_{n}\right)\right]^{1-\rho}\right. \\
\omega\left(\sigma_{n}, \sigma_{n-1}\right) q p_{b}\left(\sigma_{n+1}, \sigma_{n}\right) \leq \delta^{\frac{1}{1-\beta}}\left[\left[\frac{1}{s^{2}} q p_{b}\left(\sigma_{n-1}, \sigma_{n}\right)\right]\right. \\
\omega\left(\sigma_{n}, \sigma_{n-1}\right) q p_{b}\left(\sigma_{n+1}, \sigma_{n}\right) \leq \delta q p_{b}\left(\sigma_{n-1}, \sigma_{n}\right) \leq \varphi^{n} q p_{b}\left(\sigma_{0}, \sigma_{1}\right)
\end{gathered}
$$

So putting $n \rightarrow \infty$ in equation 3.2, we get $L=0$.

Now, to show $\sigma_{n}$ is Cauchy sequence.

Let $n, t \in N$.

$$
\begin{gathered}
q p_{b}\left(\sigma_{n}, \sigma_{n+t}\right) \leq s q p_{b}\left(\sigma_{n}, \sigma_{n+1}\right)+s^{2} q p_{b}\left(\sigma_{n+1}, \sigma_{n+2}\right)+\ldots+s^{t} q p_{b}\left(\sigma_{n+t-1}, \sigma_{n+t}\right) \\
\leq\left[s \delta^{n}+s^{2} \delta^{n+1}+\ldots+s^{t} \delta^{n+t-1}\right] q p_{b}\left(\sigma_{0}, \sigma_{1}\right) \\
\leq s^{t} \sum_{i=n}^{n+t-1} \delta^{i} q p_{b}\left(\sigma_{0}, \sigma_{1}\right) \leq s^{t} \sum_{i=n}^{\infty} \delta^{i} q p_{b}\left(\sigma_{0}, \sigma_{1}\right) \cdots
\end{gathered}
$$

From equation 3.3,

$$
\left.\lim _{n \rightarrow \infty} q p_{b}\left(\sigma_{n}, \sigma_{n+t}\right)=\lim _{m \rightarrow \infty, n \rightarrow \infty} q p_{b}\left(\sigma_{n+m}\right), \sigma_{n+m+t}\right) \leq s^{t} \lim _{m \rightarrow \infty} \sum_{i=m}^{\infty} \lim _{n \rightarrow \infty} \delta^{i} q p_{b}\left(\sigma_{n}, \sigma_{n+1}\right)=0
$$

Now, if $\sigma_{n} \neq H \sigma_{n}$.

$$
\begin{aligned}
q p_{b}\left(\sigma_{n+1}, H \eta\right)= & q p_{b}\left(H \sigma_{n}, H \eta\right) \leq \delta\left[q p_{b}\left(\sigma_{n}, H \eta\right)\right]^{\rho}\left[\frac{1}{s^{2}} q p_{b}\left(\eta, H \mu_{n}\right)\right]^{1-\rho} \\
& \leq \delta\left[q p_{b}\left(\sigma_{n}, H \eta\right)\right]^{\beta}\left[q p_{b}\left(\eta, \sigma_{n+1}\right)\right]^{1-\rho}
\end{aligned}
$$


for $\eta \in X$.

Here, for $n \rightarrow \infty, q p_{b}(\eta, H \eta)=0$.

This is a contradiction and hence $H \eta=\eta$.

Corollary 3.1 Let $\left(X, q p_{b}\right)$ be a complete quasi-partial b-metric space whose subsets $\xi_{1}$ and $\xi_{2}$ are closed. Suppose that $H: \xi_{1} \cup \xi_{2} \rightarrow \xi_{1} \cup \xi_{2}$ satisfies:

$$
\omega(\sigma, \theta) q p_{b}(H \sigma, H \theta) \leq \delta\left[q p_{b}(\sigma, H \theta)\right]^{\rho}\left[\frac{1}{s^{2}} q p_{b}(\theta, H \sigma)\right]^{1-\rho}
$$

for all $\sigma \in \xi_{1}$ and $\theta \in \xi_{2}$ such that $\sigma, \theta \in X \backslash F i x(H)$, where $\rho \geq 0, s \geq 1$. If $H\left(\xi_{1}\right) \subseteq \xi_{2}$ and $H\left(\xi_{2}\right) \subseteq \xi_{1}$, then there exists a fixed point of $\mathrm{H}$ in $\xi_{1} \cap \xi_{2}$.

Proof: In Theorem 3.1, it is enough to take, $\omega(\sigma, \theta)= \begin{cases}1 & \text { if }\left(\xi_{1} \times \xi_{2}\right) \cup\left(\xi_{2} \times \xi_{1}\right) \\ 0 & \text { otherwise }\end{cases}$

Corollary 3.2 Suppose $\left(X, q p_{b}, \preceq\right)$ be a complete partially-ordered quasi-partial b-metric space. Let $H: X \rightarrow X$ be the mapping such that:

$$
\omega(\sigma, \theta) q p_{b}(H \sigma, H \theta) \leq \delta\left[q p_{b}(\sigma, H \theta)\right]^{\rho}\left[\frac{1}{s^{2}} q p_{b}(\theta, H \sigma)\right]^{1-\rho}
$$

such that $\sigma, \theta \in X \backslash F i x(H)$ where $\rho \geq 0, s \geq 1$.

Let us assume the following:

a) $\mathrm{H}$ is non decreasing with respect to partial order $\preceq$;

b) There exists $\sigma_{0} \in X$ such that $\sigma_{0} \preceq H \sigma_{0}$;

c) $\mathrm{H}$ is continuous on $\left(\mathrm{X}, q p_{b}\right)$.

Then $\mathrm{H}$ has a fixed point in $\mathrm{X}$.

Proof: In Theorem 3.1, it is enough to take, $\omega(\sigma, \theta)= \begin{cases}1 & \text { if }(\sigma \preceq \theta) \operatorname{or}(\theta \preceq \sigma) \\ 0 & \text { otherwise }\end{cases}$

Example 3.1 : Let us consider the set $X=[0,3]$ with quasi-partial b-metric defined as $q p_{b}(\sigma, \theta)=$ $\operatorname{sink}\left|\sigma^{2}-\theta^{2}\right|$ and $\mathrm{H}$ be a self-mapping on $\mathrm{X}$ which is defined as

$H \sigma=\left\{\begin{array}{ll}\frac{5}{2} & \text { if } \sigma \in[2,3] \\ \frac{2}{7} & \text { if } \sigma \in[0,2]\end{array}\right.$ and taking, $\omega(\sigma, \theta)= \begin{cases}1 & \text { if }(\sigma, \theta) \in[2,3] \\ 0 & \text { otherwise }\end{cases}$

Let $\sigma, \theta \in X$ be such that $\sigma \neq H \sigma, \theta \neq H \theta$ and $\omega(\sigma, \theta) \geq 1 \sigma, \theta \in[2,3]$, and we have $H \sigma=H \theta=\frac{5}{2}$. Therefore, Definition 3.1 holds, for $\sigma_{0}=3$.

$\omega(3, H 3)=\omega\left(3, \frac{5}{2}\right)=1, \omega(\sigma, \theta) \geq 1$ for $\sigma, \theta \in X$. So $\sigma, \theta \in[2,3]$ and $H \sigma=H \theta \in[2,3]$.

Thus $\mathrm{H}$ is $\omega$-orbital admissible as $\omega(H \sigma, H \theta) \geq 1$.

Now we show that condition (M) holds, let us take a sequence $\theta_{n}$ in $\mathrm{X}$ such that $\omega\left(\theta_{n}, \theta_{n+1}\right) \geq 1$ for all $\mathrm{n}$, 


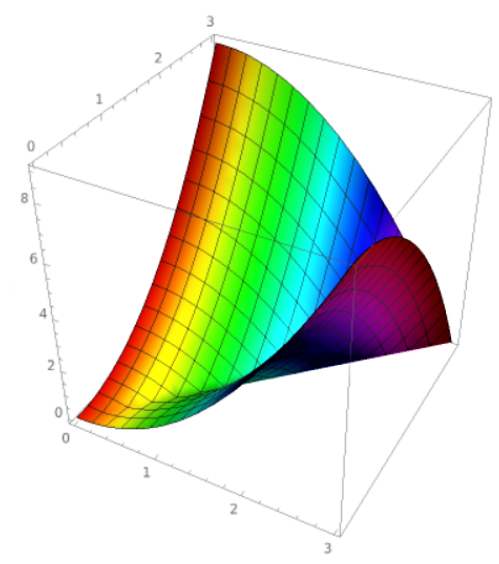

Figure 1. The fixed points of $\mathrm{H}$ are $\frac{5}{2}$ and $\frac{2}{7}$.

then $\theta_{n} \subset[2,3]$. Also as $n \rightarrow \infty, \theta_{n} \rightarrow v \in X$, we get $\left|\theta_{n}-v\right| \rightarrow 0$. Therefore $v \in[2,3], \omega\left(\theta_{n}, v\right)=1$. Hereby Theorem 3.1 holds true and the fixed points of $\mathrm{H}$ are $\frac{5}{2}$ and $\frac{2}{7}$ as shown in Figure 1.

\section{Conclusion}

The accession of this study is to commence the proposition of Interpolative Chatterjea type contraction on $\omega$-admissible mapping in quasi-partial b-metric space. $\omega$-admissibility finds it's real world applications in varying fields be it in classical game theory for finding behaviour in multi-player games or even infinite games that are played on graphs. Apart from this it is also used in deciding the extensions of DLs by concrete domains. This concept has been conceded in many researches earlier. The current research can also be exercised effectively in all these areas of study.

Acknowledgements: All authors are grateful to the Spanish Government for Grant RTI2018-094366B-I00 (MCIU/AEI/FEDER, UE) and to the Basque Government for Grant IT1207-19.

Funding: No external funding has been received in this research.

Conflicts of Interest: The author(s) declare that there are no conflicts of interest regarding the publication of this paper.

\section{REFERENCES}

[1] S. Banach, Sur les operations dans les ensembles abstraits et leur application aux equations integrales, Fund. Math. 3 (1922), 133-181.

[2] R. Kannan, Some results on fixed points-II, Amer. Math. Mon. 76 (1969), 405-408.

[3] E. Karapinar, Revisiting the Kannan Type Contractions via Interpolation, Adv. Theory Nonlinear Anal. Appl. 2 (2018), $85-87$.

[4] A. Gupta, P. Gautam, Some coupled fixed point theorems on quasi-partial b-metric spaces, Int. J. Math. Anal. 9 (2015), 293-306. 
[5] I.A. Bakhtin, The contraction principle in quasi metric spaces, Funct. Anal. 30 (1989), 26-37.

[6] S. Czerwik, Contraction mappings in b-metric spaces, Acta Math. Inform. Univ. Ostrav. 1 (1993), 5-11.

[7] S.G. Matthews, Partial metric topology, Ann. N. Y. Acad. Sci. 728 (1994), 183-197.

[8] S.K. Chatterjea, Fixed-point theorems, C. R. Acad. Bulg. Sci. 25 (1972), 727-730.

[9] P. Gautam, V.N. Mishra, R. Ali, S. Verma, Interpolative Chatterjea and cyclic Chatterjea contraction on quasi-partial b-metric space, AIMS Math. 6 (2020), 1727-1742.

[10] O. Popescu, Some new fixed point theorems for $\alpha$-Geraghty contraction type maps in metric spaces, Fixed Point Theory Appl. 2014 (2014), 190.

[11] E. Karapınar, I.M. Erhan, A. Öztürk, Fixed point theorems on quasi-partial metric spaces, Math. Comput. Model. 57 (2013), 2442-2448.

[12] A. Gupta, P. Gautam, Quasi-partial b-metric spaces and some related fixed point theorems, Fixed Point Theory Appl. 2015 (2015), 18.

[13] V.N. Mishra, L.M. Sánchez Ruiz, P. Gautam, S. Verma, Interpolative Reich-Rus-Ćirić and Hardy-Rogers Contraction on Quasi-Partial b-Metric Space and Related Fixed Point Results, Mathematics. 8 (2020), 1598.

[14] P. Gautam, L.M. Sánchez Ruiz, S. Verma, Fixed Point of Interpolative Rus-Reich-Ćirić Contraction Mapping on Rectangular Quasi-Partial b-Metric Space, Symmetry. 13 (2021), 32.

[15] P. Gautam, S. Verma, Fixed point via implicit contraction mapping on quasi-partial b-metric space, J. Anal. (2021). https://doi.org/10.1007/s41478-021-00309-6. 